\title{
Guideline Adherence and Associated Outcomes in the Treatment of Type 2 Diabetes Mellitus Patients With an Incident Cardiovascular Comorbidity: An Analysis Based on a Large German Claims Dataset
}

\author{
Maximilian Gabler · Nils Picker · Silke Geier • Ludwin Ley • \\ Jens Aberle · Michael Lehrke · Stephan Martin • Matthias Riedl • \\ Thomas Wilke
}

Received: November 27, 2020 / Accepted: February 4, 2021 / Published online: March 12, 2021

(C) The Author(s) 2021

\section{ABSTRACT}

Introduction: According to current guidelines, appropriate drug treatment is the backbone of the effective management of cardiovascular (CV) comorbidities in patients with type 2 diabetes mellitus (T2DM). The main objective of this study was to assess the degree of real-world adherence to these guideline recommendations and to identify whether poor guideline adherence is associated with worse clinical outcomes. Methods: In this retrospective German claims data analysis (AOK PLUS dataset), patients with T2DM with an incident diagnosis (index date) of ischemic stroke, myocardial infarction, heart

Supplementary Information The online version contains supplementary material available at https:// doi.org/10.1007/s13300-021-01024-y.

M. Gabler · S. Geier · L. Ley

Boehringer Ingelheim Pharma GmbH \& Co. KG, Binger Str. 173, 55216 Ingelheim am Rhein,

Germany

N. Picker $(\bowtie)$

Ingress-Health HWM GmbH, Alter Holzhafen 19,

23966 Wismar, Germany

e-mail: nils.picker@ingress-health.com

J. Aberle

Universitätsklinikum Hamburg-Eppendorf (UKE),

Martinistr. 52, 20246 Hamburg, Germany

M. Lehrke

Universitätsklinikum Aachen (RWTH),

Pauwelsstraße 30, 52074 Aachen, Germany failure or coronary artery disease were observed for 12 months between 1 January 2014 and 31 December 2017. We assessed guideline adherence per observed $\mathrm{CV}$ disease combination at three levels: "green" if patients received prescriptions of all recommended medications with $>185$ defined daily doses (DDDs) per observed patient-year; "yellow" if patients received at least two prescriptions of at least one of the recommended medications; and "red" if patients did not receive at least two prescriptions of at least one of the recommended medications. The impact of the assignment of a patient to one of these three levels on all-cause mortality and CV risk was analyzed based on multivariable Cox regression analyses and reported as adjusted hazard ratios (HRs).

Results: We identified 32,916 patients with T2DM with an incident CV comorbidity (mean

\section{S. Martin}

Westdeutsches Diabetes- und Gesundheitszentrum (WDGZ), Hohensandweg 37, 40591 Düsseldorf, Germany

M. Riedl

Medicum Hamburg MVZ GmbH, Beim Strohhause

2, 20097 Hamburg, Germany

T. Wilke

Institut für Pharmakoökonomie und

Arzneimittellogistik (IPAM), University of Wismar, Alter Holzhafen 19, 23966 Wismar, Germany 
age 75.0 years, $54.2 \%$ female, Charlson Comorbidity Index [CCI]: 5.5). Observed patients received at least 185 DDDs of the following medication classes in the 12 months before/after the index date: vitamin $\mathrm{K}$ antagonists $(6 \% / 6 \%)$; antiplatelet drugs (9\%/27\%); novel oral anticoagulants (3\%/13\%); diuretics (48\%/54\%); beta blockers (31\%/35\%); calcium-channel blockers (34\%/32\%); renin-angiotensin-aldosterone system inhibitors (69\%/68\%); and lipid-modifying agents $(19 \% / 37 \%)$. When post-index therapy was compared to guideline recommendations, the level of "guideline adherence" was classified as "green" for $14.4 \%$ of the patients, "yellow" for $75.2 \%$ and "red" for $10.5 \%$. An assignment of "red" was associated with worse CV outcomes in all analyses. Regarding mortality, in addition to one additional year of age (hazard ratio [HR] 1.04), CCI (HR 1.17), use of insulins (HR 1.25), digitalis glycosides (HR 1.52) and diuretics (HR 1.32), non-adherence to guideline recommendations ("red": HR 6.79; "yellow": HR: 1.30) was a significant predictor for early death, while female gender (HR 0.79), the participation in a disease management program (HR 0.69) and the use of antidiabetics other than insulin (HR 0.74) were generally associated with a reduced risk.

Conclusion: Only a minority of patients with T2DM and an incident CV comorbidity receive a treatment fully adherent with guideline recommendations. This may contribute to high mortality rates in this population in clinical practice.

Keywords: Cardiovascular disease; Cardiovascular drug treatment; Guideline adherence; T2DM

\section{Key Summary Points}

\section{Background}

Most patients with type 2 diabetes (T2DM)

suffer from multiple additional comorbidities, especially cardiovascular (CV) diseases such as coronary heart disease and heart failure, or have experienced previous events, such as myocardial infarction or stroke.
Less is known about the long-term CV treatment and treatment-associated outcomes of these patients, as real-world evidence studies mostly focus on one specific disease and associated outcomes.

Current clinical guidelines recommend an appropriate management of $\mathrm{CV}$ comorbidities with cardiovascular medications.

\section{Study objectives}

Description of the real-life medication of patients with T2DM who suffer from at least one incident $\mathrm{CV}$ comorbidity.

Comparison of treatment patterns of these patients with guideline recommendations for prevention of subsequent CV events.

Evaluation of impact of guideline nonadherence on the CV event risk, hospitalization rates and mortality risk.

\section{Key learning points}

Drug treatment of a substantial proportion of patients with T2DM with an incident CV comorbidity did not follow guideline recommendations for prevention of subsequent $\mathrm{CV}$ events.

In result, mortality risk and CV event rates were found to be considerably higher in this patient cohort than in respective clinical trials.

We strongly recommend that the drug treatment of this population be more closely monitored and that guidelineadherent treatment included as an indicator of be quality management in healthcare.

\section{DIGITAL FEATURES}

This article is published with digital features, including a summary slide, to facilitate understanding of the article. To view digital features 
for this article go to https://doi.org/10.6084/ m9.figshare.13713529.

\section{INTRODUCTION}

Type 2 diabetes mellitus (T2DM) is one of the most common chronic diseases in the world. According to the International Diabetes Federation atlas, the prevalence of diabetes in 2019 was estimated to be $9.3 \%$ (463 million people). The prevalence of this disease continues to rise across the globe, while health expenditures and mortality rates related to diabetes continue to have an increasing social and financial impact on modern healthcare systems. For 2045, the prevalence of diabetes in adults is estimated to be as high as $10.9 \%$ (700 million) [1].

Diabetic patients face an increased risk of micro- and macrovascular complications and increased mortality rates [2]. Depending on the exact definition of the comorbidity, approximately $30-45 \%$ of people with T2DM additionally suffer from cardiovascular disease (CVD), with the percentage increasing with higher age [3]. CVD and cardiovascular (CV) events such as stroke, myocardial infarction (MI) or heart failure (HF) are the leading cause of death in patients with T2DM, leading to a life expectancy of patients with diabetes that is 10 years shorter than that of patients without diabetes [4].

There is an urgent need for an effective management of patients with T2DM who also suffer from CVD. Current clinical guidelines recommend-in addition to an appropriate management of diabetes, specifically avoiding hypoglycemia and weight gain and ensuring good glucose control [5]-an appropriate management of $\mathrm{CV}$ comorbidities with cardiovascular medications [6-10].

Data on the real-world (RW) treatment for CVDs in patients with T2DM is rare $[11,12]$. Therefore, the objective of this study was to fill that data gap by describing the RW treatment of patients with T2DM and an incident CVD, and to compare the observed drug treatment of these patients with the respective clinical guidelines. We also assessed the impact of guideline non-adherence on the risk of a $\mathrm{CV}$ event, hospitalization rates and mortality risk.

\section{METHODS}

\section{Dataset}

This study was a retrospective claims data analysis using an anonymized database provided by a German statutory health insurance fund (AOK PLUS). This claims database includes about 3.1 million insured persons in Germany (Saxonia, Thuringia), which reflects $4.3 \%$ of the German population insured by statutory health insurance funds. Generally, German claims data contain information on patients' demographics (age, gender, date of death) and detailed reimbursement-related data on outpatient care, inpatient care, pharmaceuticals, therapeutic devices, rehabilitation and sick leaves. Specifically, for outpatient medication prescriptions the following information is available: agent class (according to the Anatomical Therapeutic Chemical [ATC] classification system) and related defined daily dose (DDD) as published by the German Institute of Medical Documentation and Information (DIMDI) [13], date of prescription/filling in the pharmacy, the prescribed package size, the route of administration and the strength of the prescribed agent.

\section{Study Population and Observational Period}

We included individuals who were continuously insured in the study period from 1 January 2013 to 31 December 2017 or until the date of death. A diagnosis of existing T2DM was required to be confirmed by at least two outpatient diagnoses by a specialist physician (excluding diagnoses from general practitioners) or one inpatient T2DM diagnosis (International statistical classification of diseases and related health problems, 10th revision, German modification [ICD-10 GM]: E11). Patients with a documented concomitant T1DM diagnosis (ICD-10 GM: E10) during the study period were excluded from the analysis. 
Of this selected population with T2DM, we identified patients with an incident $\mathrm{CV}$ event/diagnosis in the inclusion period between 1 January 2014 and 31 December 2016, ensuring a minimum pre- and post-index period of 12 months each. The date of the observed incident CV event/diagnosis was defined as the study index date. Generally, the following $\mathrm{CV}$ event/disease categories were taken into account: ischemic stroke (IS; ICD-10 code: I63), MI (ICD-10 code: I21), HF (ICD-10 codes: I11.0, I13.0, I13.2, I50) and coronary artery disease (CAD; ICD-10 code: I25). In the case of IS or MI, we required at least one respective inpatient diagnosis; in the case of $\mathrm{HF}$ and CAD, two confirmed outpatient diagnoses documented by a cardiologist were also accepted as proof of the existence of that disease. To ensure that we observed only patients with an incident CVD, patients with any diagnosis for one of the following pre-specified CVDs documented in the pre-index period (minimum of 12 months) were excluded: all types of stroke (ICD-10 codes: I63, I64, I69.3 or I69.4; only for inpatient setting), MI (ICD-10 codes: I21, I22 or I23; only for inpatient setting), HF (ICD-10 codes: I11.0, I13.0, I13.2, I50, or I51) and CAD (ICD-10 codes: I24 or I25).

Patients could be assigned to more than one group of CV disease (IS, MI, HF, CAD) if-at the index date-more than one of these diseases was diagnosed. Patients aged younger than 18 years at the index date were excluded from the analysis.

Patients were observed from their incident diagnosis of a CV disease for 12 months or until death, whatever came first. However, analysis of medication treatment and its comparison with guideline recommendations was only conducted for those who survived the first 90 days since their incident CVD diagnosis, to ensure that treatment patterns could be observed for a sufficient time.

Patient characteristics were described separately per subgroup according to their incident CVD based on a 12-months pre-index period. Reported baseline characteristics included age, gender, and comorbidities, which were described using the adapted Diabetes Complications Severity Index (aDCSI; Electronic
Supplementary Material [ESM] Table 1) [14] and the Charlson Comorbidity Index (CCI; ESM Table 2) [15].

\section{Description of RW Treatment of Patients with T2DM with an Incident CVD}

The pharmacological treatment of target patients was described based on documented outpatient prescriptions in the 12 months before and 12 months after the index date (= incident CVD diagnosis). In both the preand post-index periods, prescriptions of antidiabetic drugs (AD; ATC code: A10*), antithrombotic agents (ATC code: B01*), diuretics (ATC code: $\mathrm{C} 01^{*}$ ), beta-blocking agents (BB; ATC code: $\left.\mathrm{CO3}^{*}\right)$, calcium channel blockers (CCB; ATC code: $\mathrm{C}^{*}{ }^{*}$, renin-angiotensin-aldosterone system acting agents (RAAS-acting agents; ATC code: C09*) and lipid-lowering therapies (LLT; ATC code: $\mathrm{C} 10^{\star}$ ) were analyzed (ESM Table 3).

\section{Evaluation of Guideline Adherence in the Treatment of Patients with T2DM with an Incident CVD}

Drug treatment for $\mathrm{CV}$ comorbidities, as observed within the 12 months after the index date, was compared to the recommendations of known clinical guidelines for each specific subgroup. The following CV guidelines were utilized: (1) S3 Leitlinie Sekundärprophylaxe ischämischer Schlaganfall und transitorische ischämische Attacke (S3 Guideline "Secondary prevention of ischemic stroke and transitory ischemic attack" [10] for IS; (2) Pocket-Leitlinie: Therapie des akuten Herzinfarktes bei Patienten mit ST-Streckenhebung (STEMI) (Pocket Guideline "Therapy of ST-elevation myocardial infarction") [7] as well as the Pocket-Leitlinie: Akutes Koronarsyndrom ohne ST-Hebung (NSTE-ACS) (Pocket Guideline "Acute coronary syndrome without ST-elevation") [6] for MI; (3) Nationale VersorgungsLeitlinie: Chronische Herzinsuffizienz [8] ("National treatment guideline Chronic heart failure") for HF; and (4) Nationale VersorgungsLeitlinie: Chronische koronare Herzkrankheit ("National Treatment 
Table 1 Definition for adherence to guideline recommendations

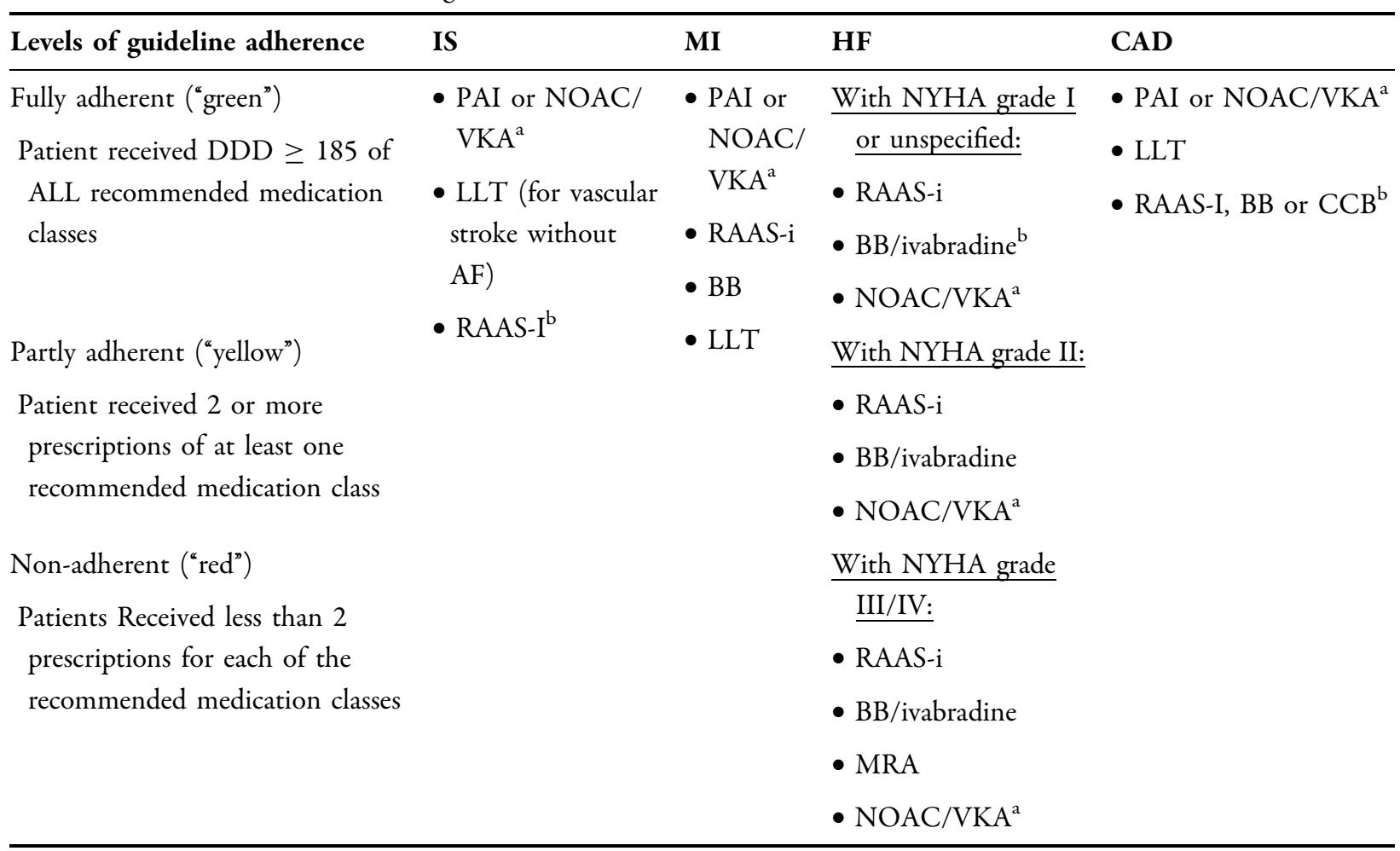

Recommendations on long-term follow-up medication are according to respective clinical guidelines in Germany $A F$ Atrial fibrillation, $B B$ beta-blocking agent, $C C B$ Calcium-channel blocker, $C A D$ coronary artery disease, $D D D$ daily defined dose, $H F$ heart failure, $I S$ ischemic stroke, $L L T$ lipid-lowering therapy, $M I$ myocardial infarction, $M R A$ mineralocorticoid receptor/aldosterone antagonist, $N O A C$ non-vitamin-K antagonist oral anticoagulant, $P A I$ platelet-aggregation inhibitor, $R A A S-I$ renin-angiotensin-aldosterone system inhibitor, $V K A$ vitamin $\mathrm{K}$ antagonist

${ }^{a}$ Use of VKA/NOAC was considered as compliant to guideline recommendations only, if a present AF was confirmed based on at least 1 documented inpatient or outpatient diagnosis with ICD-10 code I48

${ }^{b}$ Use of additional medication to lower blood pressure was considered as compliant to guideline recommendations only, if existing hypertension was confirmed based on at least 1 documented inpatient or outpatient diagnosis ICD-10 code I10-I15

Guideline Chronic coronary heart disease") [9] for CAD.

Adherence to guideline-recommended therapies is outlined in Table 1. Guideline adherence per observed $\mathrm{CV}$ disease combination was assessed at three levels. A patient who received prescriptions for all recommended medications with $>185$ defined daily doses (DDDs) per observed patient-year was assessed to be level "green" (indicating full guideline adherence). Patients who received at least two prescriptions for at least one of the recommended medications were assessed to be level "yellow" (indicating partial agreement with guideline recommendations). Finally, patients who did not receive at least two prescriptions for at least one of the recommended medications were assessed to be level "red" (indicating no agreement with guideline recommendations). In the case of prescribed fixed-dose combinations, both corresponding medication groups were acknowledged as prescribed agents.

\section{Description of Clinical Outcomes}

In addition to all-cause hospitalizations and allcause death, acute hospitalization with the following primary/secondary diagnoses (all ICD10 codes) have been considered as relevant events: all-cause stroke (I60, I61, I62, I63 or I64), MI (I21), HF (I11.0, I13.0, I13.2, or I50), 
unstable angina pectoris (I20.0), CAD (I25), transient ischemic attack (G45), arterial embolism (H34, I26 or K55.0), peripheral vascular disease (A48, E11.5, I73.9, I74.3, L97, R02 or S91), peripheral artery disease (I70.2), hypoglycemia (E16.2-), coronary revascularizations (procedure [OPS] codes: $5-361,5-362$ or $5-363$ ), as well as percutaneous transluminal vascular interventions and stent implantations (OPS 8-836/8-837/8-84). In accordance with the recent literature on this topic [16-21], two composite CV endpoints were defined: any inpatient diagnosis for HF (I11.0, I13.0, I13.2, or I50) or all-cause death (endpoint CV-2) and any inpatient diagnosis for MI (I21) or stroke (I6064) or all-cause death (endpoint CV-3).

\section{Statistical Analysis}

All variables were descriptively analyzed by means of summary statistics (mean, standard deviation [SD]) for continuous data and frequency tables for categorical data.

Time to first post-index hospitalization events was depicted using Kaplan-Meier (KM) curves for pre-specified patient subgroups: by index event (IS, MI, HF or CAD) or, for patients included in the guideline-adherence analysis, by the level of agreement with guidelines (green-yellow-red). Restricted means for the event-free time were reported if the median was not reached. The significance of differences of time to events was tested by using log-rank (Mantel-Cox) tests.

To adjust for differences in patient characteristics between compared subgroups, guideline adherence and other risk factors associated with the time-to-event were analyzed by multivariate Cox regression models based on patient-individual follow-up times. The following risk factors with respect to all-cause hospitalization, mortality and composite CV-related endpoints (CV-2 and CV-3) were also evaluated: age, gender, comorbidity (by CCI and aDCSI), participation in disease management programs (DMPs), previous insulin therapy, previous treatment with other ADs and previous use of digitalis glycosides and/or diuretics.
Data processing and statistical analyses were performed using MS Excel (2016 version; Microsoft Corp., Redmond, WA, USA), Stata (version 14.1; StataCorp LLC, College Station, TX, USA) and MySQL (version 8.0; open source).

\section{Ethical compliance}

Because of the non-interventional, retrospective nature of this study and because our analysis involved an anonymized dataset, neither ethical review nor patients' informed consent was required, in accordance with German law and policies of the institutions assessing patientlevel data (IPAM and AOK PLUS). The study protocol was reviewed and approved by the sickness fund (AOK PLUS) before access to the data was granted. The study was also evaluated by a scientific steering committee to which all the authors belonged and was based on a study protocol approved before the start of data analysis.

\section{RESULTS}

\section{Patient Characteristics}

From the 455,489 patients with T2DM with records in the database, we identified 120,383 patients with T2DM with at least one diagnosis of IS, MI, HF, and/or CAD (26.4\% of the T2DM population registered in the database). Among these 120,383 patients, 32,916 (7.2\%) received their incident diagnosis of the targeted CVD between 1 January 2014 and 31 December 2016: 11,427 patients in 2014, 11,122 in 2015 and 10,367 in 2016. Incident HF cases represented the largest subgroup ( $n=20,969$ [67.7\%]), followed by incident CAD cases $(n=11,275$ $[34.3 \%]), \quad$ IS $(n=5321 \quad[16.2 \%])$ and MI $(n=3557$ [10.8\%]). It should be noted that patients could be assigned to more than one diagnosis group when there were more than one of these diagnoses at the index date.

Table 2 provides the baseline characteristics for all patients as well as per subgroup. The mean age of the included patients was 75.0 years (in MI or CAD patients: 73 years; in 
Table 2 Baseline characteristics of patients with type 2 diabetes mellitus with an incident cardiovascular disease

\begin{tabular}{llllll}
\hline Baseline characteristics $^{\mathbf{a}}$ & Overall & IS & MI & HF & CAD \\
\hline$N(\%)$ & $32,916(100.0 \%)$ & $5321(16.2 \%)$ & $3557(10.8 \%)$ & $20,969(63.7 \%)$ & $11,275(34.3 \%)$ \\
Age (years) & $75.0 \pm 11.0$ & $75.0 \pm 10.9$ & $72.9 \pm 11.8$ & $76.0 \pm 10.9$ & $72.9 \pm 10.8$ \\
Female gender & $17,850(54.2 \%)$ & $2806(52.7 \%)$ & $1571(44.2 \%)$ & $12,035(57.4 \%)$ & $5144(45.6 \%)$ \\
aDCSI & $4.0 \pm 1.9$ & $4.3 \pm 1.9$ & $4.3 \pm 1.6$ & $4.1 \pm 1.9$ & $3.9 \pm 1.9$ \\
CCI & $5.5 \pm 2.6$ & $5.6 \pm 2.5$ & $5.2 \pm 2.5$ & $5.8 \pm 2.7$ & $5.2 \pm 2.4$ \\
Kidney disease & $8108(24.6 \%)$ & $947(17.8 \%)$ & $717(20.1 \%)$ & $5968(28.5 \%)$ & $2276(20.2 \%)$ \\
Hypertension & $4617(86.8 \%)$ & $2988(84.0 \%)$ & $19,151(91.3 \%)$ & $10,116(89.7 \%)$ & $4617(86.8 \%)$ \\
Diagnosed in 2014 & $11,427(34.7 \%)$ & $1791(33.7 \%)$ & $1168(32.8 \%)$ & $7224(34.4 \%)$ & $3988(35.4 \%)$ \\
Diagnosed in 2015) & $11,122(33.8 \%)$ & $1783(33.5 \%)$ & $1216(34.2 \%)$ & $7095(33.8 \%)$ & $3802(33.7 \%)$ \\
Diagnosed in 2016 & $10,367(31.5 \%)$ & $1747(32.8 \%)$ & $1173(33.0 \%)$ & $6650(31.7 \%)$ & $3485(30.9 \%)$ \\
\hline
\end{tabular}

Values in table are presented as the mean \pm standard deviation $(\mathrm{SD})$ or as a number with the percentage in parenthesis aDCSI adapted Diabetes Complications Severity Index, CCI Charlson Comorbidity Index

a Baseline characteristics of patients with T2DM with incident diagnosis of IS, MI, HF and CAD. aDCSI and CCI measured patients' comorbidity status; their calculation is based on observed diagnosed comorbidities within the 12 months prior to index (incl. index date). Frequencies of kidney disease (ICD 10: I12-13, N17-N19 or P96.0) and hypertension (ICD 10: I10-I15) were based on observed diagnoses within the 12 months prior to index

HF patients: 76 years). Of the observed patients, $54.2 \%$ were female (minimum with MI: $44.2 \%$; maximum with HF: $57.4 \%)$. Mean CCI was 5.5, and mean aDCSI was 4.0 in the whole patient sample. The vast majority of patients had been previously diagnosed with hypertension $(n=29,750$ [90.4\%]), and almost one in four patients suffered from reduced kidney function $(n=8108$ [24.6\%]), identified by ICD-10 diagnoses (ICD-10 codes for hypertension: I10-I15; kidney disease: I12-13, N17-N19 or P96.0).

\section{Treatment Patterns}

As presented in Fig. 1, treatment patterns with observed $\mathrm{AD}$ and $\mathrm{CV}$ agents did not significantly change when compared between the 12-month baseline period prior to the index date and the 12-month follow-up period. Lipidlowering therapies (LLT) and anticoagulative therapies with platelet aggregation inhibitors (PAIs) and/or non-vitamin-K antagonist oral anticoagulants (NOACs) represented the only notable exception from this, as the percentage of patients treated with these agents increased markedly (LLTs: +18 percentage points, PAIs: +18 percentage points and NOACs: +10 percentage points). Regarding AD therapy, the most frequently observed medication groups were metformin, insulins, dipeptidyl peptidase4 (DPP-4) inhibitors and sulfonylureas, while other AD regimens, such as sodium-glucose cotransporter-2 inhibitors (SGLT-2is) or glucagon-like peptide-1 receptor agonists (GLP1RAs), constituted a minority of the observed medication groups. In addition, a notable number of patients were not receiving any AD prescription within 12 months prior to/post index event $(43 \% / 47 \%)$.

The most frequently prescribed $\mathrm{CV}$ agents with at least 185 DDDs per patient-year during the 12-month baseline and follow-up periods, respectively, were RAAS inhibitors $(69 \% / 68 \%$ of patients treated), diuretics (48\%/54\%) and BBs (31\%/35\%), CCBs $(34 \% / 32 \%)$ and LLTs $(19 \% /$ $37 \%)$. Based on an additional sensitivity analysis that defined treated patients less restrictively, taking into consideration all patients 


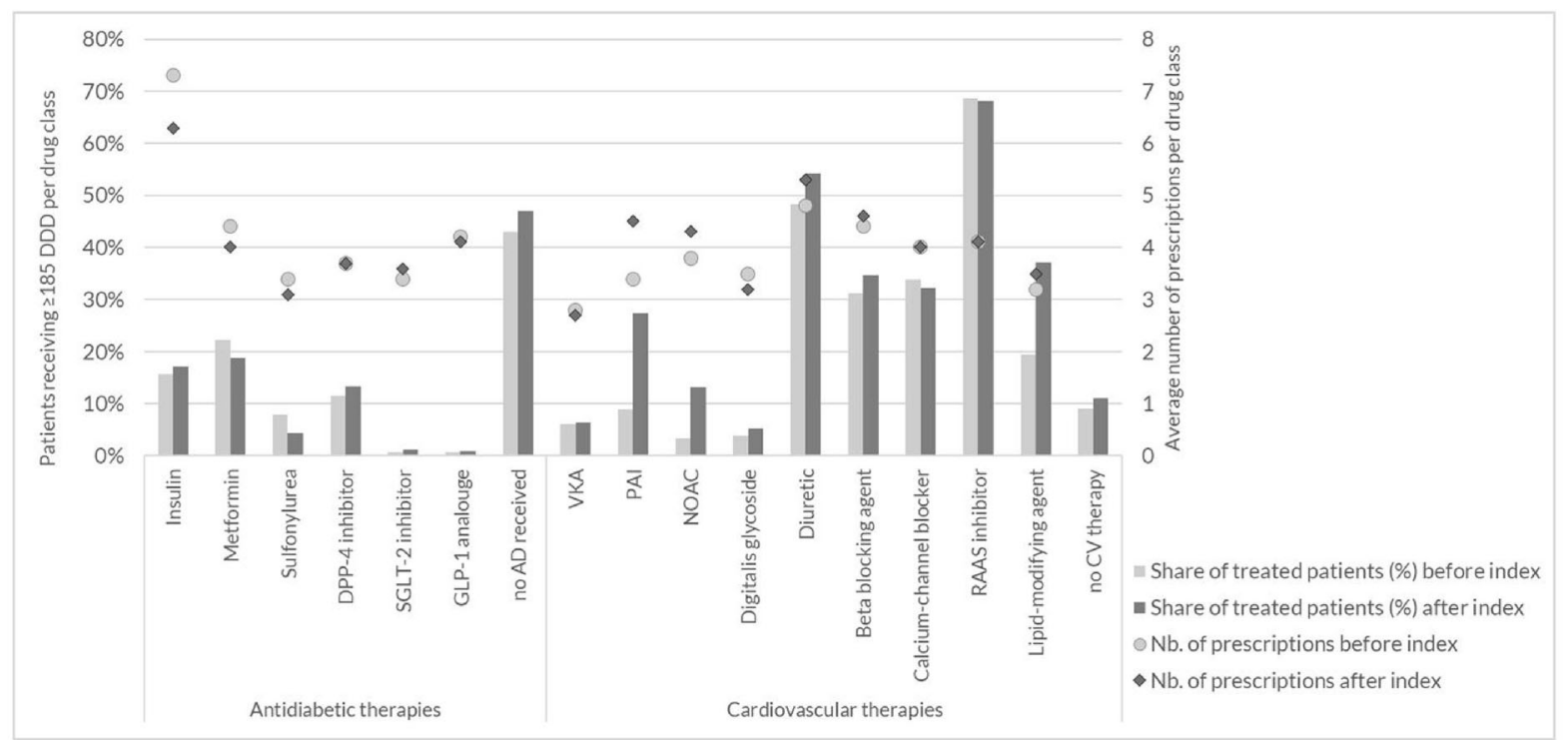

Treatments were described for observed $A D$ and $C V$ agents for patients who received $\geq 185$ DDD per patient year of the respective medication class. $\mathrm{AD}=$ antidiabetic drugs; $\mathrm{CV}=$ cardiovascular; $\mathrm{DDD}=$ defined daily dose; $\mathrm{VKA}=$ vitamin $\mathrm{K}$ antagonist; $\mathrm{PAI}=$ platelet aggregation inhibitors; $\mathrm{NOAC}=\mathrm{Non}-$ VKA anticoagulants; RAAS $=$ Renin-Angiotensin-Aldosterone-System

Fig. 1 Treatment patterns within 12 months before and after the incident $\mathrm{CV}$ diagnosis. Treatment patterns were described for observed $\mathrm{AD}$ and $\mathrm{CV}$ agents for patients who received at least 185 DDDs per patient year of the respective medication class. $A D$ Antidiabetic drugs, $C V$ cardiovascular, $D D D$ defined daily dose, $D D P-4$ dipeptidyl

with at least two prescriptions of an agent regardless of the received dose, patients were treated-in the pre-index/post-index periods with RAAS inhibitors $(67 / 64 \%)$, diuretics $(54 \% /$ $59 \%)$, BBs (48\%/55\%), CCBs (34\%/31\%) and LLTs $(23 \% / 39 \%)$. In total, throughout the whole baseline and follow-up period, 9 and $11 \%$ of patients, respectively, did not receive any CVrelated outpatient drug prescription.

\section{Adherence to CV Guidelines}

Of the 32,916 patients with diabetes who received their incident diagnosis of the targeted CVD between 1 January 2014 and 31 December $2016,28,456$ (86.5\%) who had been continuously observed for at least 90 days after the index date were included in the analysis of adherence to clinical guideline recommendations. Only $14.4 \%$ of these patients could be labeled as "green" based on receiving at least 185 DDDs of each of the recommended agents peptidase-4, GLP-1RA glucagon-like peptide-1 receptor agonists, $N O A C$ non-VKA anticoagulants, $P A I$ platelet aggregation inhibitors, $R A A S$ renin-angiotensin-aldosterone system, $S G L T-2$ sodium-glucose cotransporter-2, $V K A$ vitamin $\mathrm{K}$ antagonists

per observed patient-year, $75.2 \%$ could be labeled as "yellow" because they received at least two prescriptions of at least one of the recommended agents and for $10.5 \%$ there was no agreement with guidelines (labeled "red") as they did not receive at least two prescriptions of any of the recommended agents.

With respect to an incident IS, 1949 (42.6\%) patients with IS could be classified as "green" as they received $\geq 185$ DDDs per patient-year of each recommended agent, 2277 (49.8\%) were labeled as "yellow" as they received at least two prescriptions of at least one of the recommended agents and 346 (7.6\%) patients did not receive more than one prescription of any of the recommended agents and were labeled "red".

In contrast, considerably fewer patients with an incident MI, HF or CAD were determined to be treated in line with the respective guideline recommendations. Treatment of only $21.2 \%$ (MI), $6.1 \%(\mathrm{HF})$ and $15.2 \%$ (CAD) of these patients was found to fully comply with clinical 
recommendations ("green"), while treatment of the vast majority of these patients was classified as "yellow" (75.8, 82.7 and $76.7 \%$, respectively). The highest proportion of "red" patients was observed for HF (11.2\%) and CAD (8.1\%), while patients who experienced an acute MI as incident CV event rarely fell into this group (2.9\%).

Patient baseline characteristics by level of guideline adherence are reported in Table 3 . The mean age of patients assigned to the "red" group was substantially higher (75.6 years) than that of patients assigned to the "green" group (71.9 years). In addition, the former exhibited a higher CCI score (5.8 vs. 4.9) and women accounted for a considerably higher proportion (54.4 vs. $47.5 \%)$ than for those whose treatment was classified as "green".

An observed diagnosis of kidney disease, which is a contraindication for selected CV drugs, was less frequently observed in patients classified as "green" versus those labeled "red" (17.5 vs. $24.6 \%)$. However, even in patients without any diagnosis for kidney disease, the percentage of patients assigned to the "red" and "yellow" groups was 10.3 and $74.1 \%$, respectively, matching the 10.5 and $75.2 \%$ for the overall population.

\section{Clinical Outcomes}

Within the follow-up period of 12 months after the incident CV diagnosis, we observed 2.7 allcause (re-)hospitalizations and 26.1 hospitalization days per patient-year. The most frequent $\mathrm{CV}$-related diagnoses/procedures causing these admissions were HF (28\%), CAD (19\%), stroke $(6 \%)$, acute MI (6\%) and stent-related procedures $(6 \%)$.

In the follow-up period, $22.1 \%$ of observed patients $(n=7273)$ died. One-year mortality was highest in patients with MI (956 patients [26.9\%]), followed by HF (5356 [25.5\%]) and IS (1215 [22.8\%]), while a substantially smaller proportion of patients with an incident CAD diagnosis (1633 [14.0\%]) died during the first year of follow-up. Based on the sample of 28,456 patients who were considered for the guideline adherence analysis and who survived the first 3 months after their incident CV
Table 3 Characteristics of patients assigned to different guideline adherence subgroups

\begin{tabular}{|c|c|c|c|}
\hline \multirow{2}{*}{$\begin{array}{l}\text { Patient } \\
\text { characteristics }\end{array}$} & \multicolumn{3}{|c|}{ Guideline adherence subgroups ${ }^{b}$} \\
\hline & $\begin{array}{l}\text { "Green" } \\
\text { level of } \\
\text { guideline } \\
\text { adherence }\end{array}$ & $\begin{array}{l}\text { "Yellow" } \\
\text { level of } \\
\text { guideline } \\
\text { adherence }\end{array}$ & $\begin{array}{l}\text { "Red" level } \\
\text { of } \\
\text { guideline } \\
\text { adherence }\end{array}$ \\
\hline$N(\%)$ & $\begin{array}{l}4096 \\
\quad(14.4 \%)\end{array}$ & $\begin{array}{l}21,386 \\
\quad(75.2 \%)\end{array}$ & $\begin{array}{l}2974 \\
\quad(10.5 \%)\end{array}$ \\
\hline $\begin{array}{l}\text { With incident } \\
\text { IS }\end{array}$ & $\begin{array}{l}1949 \\
(42.6 \%)\end{array}$ & $\begin{array}{l}2277 \\
\quad(49.8 \%)\end{array}$ & $346(7.6 \%)$ \\
\hline $\begin{array}{l}\text { With incident } \\
\text { MI }\end{array}$ & $\begin{array}{l}599 \\
(21.2 \%)\end{array}$ & $\begin{array}{l}2140 \\
\quad(75.8 \%)\end{array}$ & $83(2.9 \%)$ \\
\hline $\begin{array}{l}\text { With incident } \\
\text { HF }\end{array}$ & $\begin{array}{l}1982 \\
(11.2 \%)\end{array}$ & $\begin{array}{l}14,609 \\
(82.7 \%)\end{array}$ & $\begin{array}{l}1084 \\
(6.1 \%)\end{array}$ \\
\hline $\begin{array}{l}\text { With incident } \\
\text { CAD }\end{array}$ & $\begin{array}{l}1561 \\
\quad(15.2 \%)\end{array}$ & $\begin{array}{l}7898 \\
\quad(76.7 \%)\end{array}$ & $833(8.1 \%)$ \\
\hline Age (years) & $71.9 \pm 10.6$ & $74.5 \pm 10.8$ & $\begin{array}{c}75.6 \pm \\
12.1\end{array}$ \\
\hline Female gender & $\begin{array}{l}1945 \\
(47.5 \%)\end{array}$ & $\begin{array}{l}11,724 \\
\quad(54.8 \%)\end{array}$ & $\begin{array}{l}1617 \\
(54.4 \%)\end{array}$ \\
\hline CCI & $4.9 \pm 2.2$ & $5.2 \pm 2.4$ & $5.8 \pm 2.9$ \\
\hline $\mathrm{aDCSI}$ & $3.9 \pm 1.7$ & $3.9 \pm 1.9$ & $3.6 \pm 1.9$ \\
\hline Kidney disease & $\begin{array}{l}716 \\
(17.5 \%)\end{array}$ & $\begin{array}{l}5286 \\
(24.7 \%)\end{array}$ & $\begin{array}{l}732 \\
(24.6 \%)\end{array}$ \\
\hline Hypertension & $\begin{array}{l}375 \\
\quad(90.8 \%)\end{array}$ & $\begin{array}{l}1684 \\
\quad(92.1 \%)\end{array}$ & $\begin{array}{l}612 \\
(79.4 \%)\end{array}$ \\
\hline
\end{tabular}

Description of patient characteristics according to the level of guideline adherence for recommended drug therapies related to IS, MI, HF and CAD

Values in table are presented as the mean $\pm \mathrm{SD}$ or as a number with the percentage in parenthesis

a aDCSI and CCI are measures of patients' comorbidity status; their calculation is based on observed diagnosed comorbidities within the 12 months prior to index (including index date). Frequencies for patients with kidney disease (ICD 10: I12-13, N17-N19 or P96.0) and hypertension (ICD 10: I10-I15) are based on observed diagnoses within 12 months prior to index

b See Table1 and text for full description of treatment groups 
diagnosis, overall 9-month mortality was 9.9\% (4.7\% for "green" patients, $7.5 \%$ for "yellow" patients and $34.1 \%$ for "red" patients (Fig. 2).

$\mathrm{KM}$ curves for time to first observed composite $\mathrm{CV}$ endpoints (CV-2: inpatient hospitalization due to HF or all-cause death; CV-3: inpatient hospitalization due to nonfatal MI, stroke or all-cause death) are shown per indication and classified by level of adherence in Fig. 3. In comparison to patients classified as "red," those who received at least two prescriptions of at least one recommended agent ("yellow") or whose treatment was fully in line with guidelines ("green") had a significantly longer event-free time with respect to CV-2 (restricted mean for event-free time $[95 \%$ confidence interval $\{\mathrm{CI}\}]$ : fully adherent "green": 321 [318-324] days; partly adherent "yellow": 273 [272-275] days; non-adherent "red": 259 [255-265] days) and CV-3 (restricted mean: 315 [311-318] days/332 [331-333] days/287 [283-291] days; Fig. 4). This pattern was also observed in all indication-specific subgroups analyses (ESM Figs. 3, 4). Additional time-toevent analyses with respect to death and allcause hospitalizations can be found in ESM Figs. 1 and 2.

Four multivariable Cox regression analyses (Table 4) were conducted to investigate the effect of guideline adherence on $\mathrm{CV}$ and

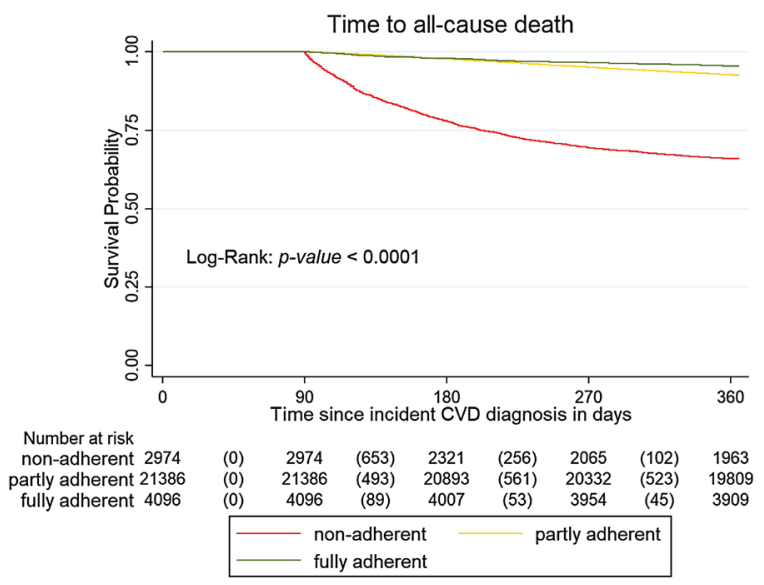

Fig. 2 Kaplan-Meier analysis: time to all-cause death. Only patients with a minimum survival time of 90 days were considered in this analysis. CVD Cardiovascular disease

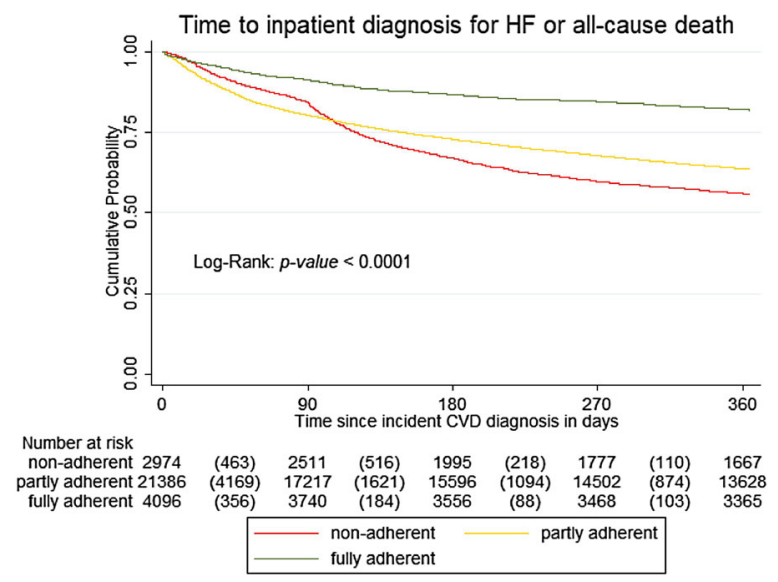

Fig. 3 Kaplan-Meier analysis: time to composite CV-2 endpoint. Patients who died within the first 90 days were excluded. Hence, only nonfatal CV events could occur during the first 90 days. CV-2 endpoint is hospitalization with heart failure or death within 12 months after index date according to indication and guideline adherence

mortality risk. With respect to all-cause mortality, one additional year of age (hazard ratio [HR] 1.04; 95\% CI 1.03-1.04), higher CCI (HR 1.17 ; $95 \%$ CI 1.15-1.18), higher aDCSI (HR 1.02; 95\% CI 1.00-1.04), use of insulins (HR 1.25; 95\% CI 1.15-1.36), digitalis glycosides (HR 1.52; 95\% CI 1.37-1.71) and diuretics (HR 1.32; 95\%

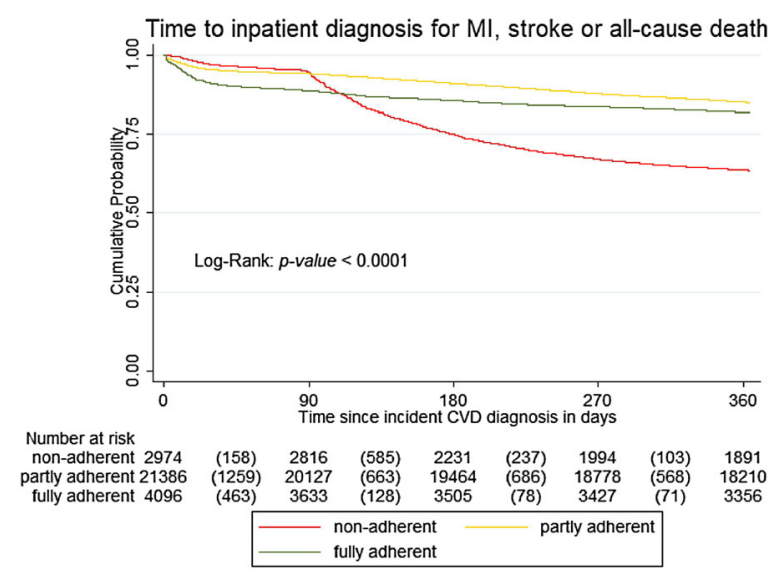

Fig. 4 Kaplan-Meier analysis: time to composite CV-3 endpoint. Patients who died within the first 90 days were excluded. Hence, only nonfatal CV events could occur during the first 90 days. CV-3 endpoint is hospitalization with myocardial infarction, stroke or death within 12 months after index according to indication and guideline adherence 


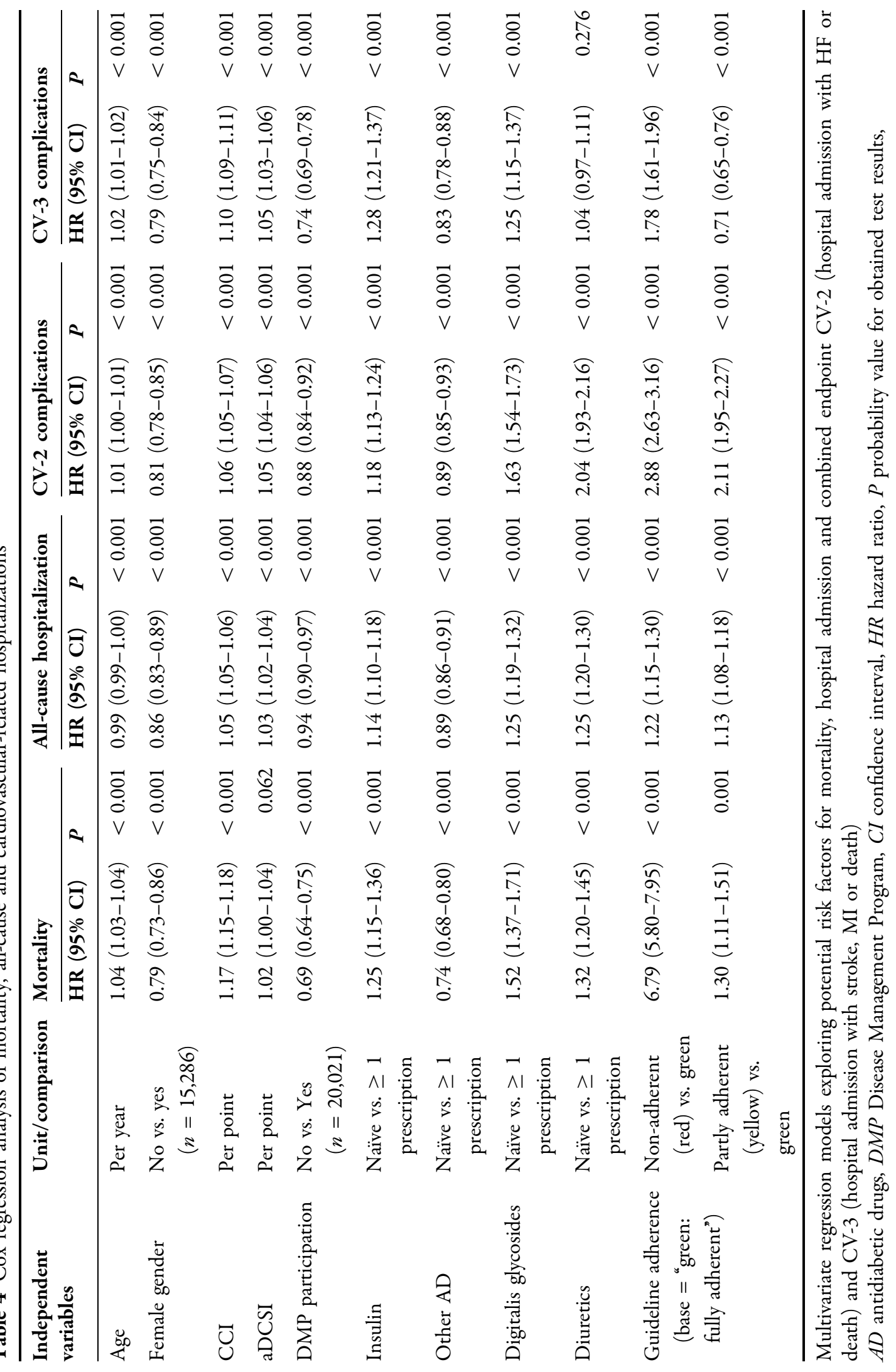


CI 1.20-1.45) as well as lack of guideline adherence (non-adherent "red": HR 6.79; 95\% CI 5.80-7.95; partly adherent "yellow": HR 1.30; 95\% CI 1.11-1.51) were associated with a higher risk of early death after 3 months post-index. Female gender (HR 0.79; 95\% CI 0.73-0.86), DMP participation (HR 0.69; 95\% CI 0.64-0.75) and use of a non-insulin AD regimen (HR 0.74; 95\% CI 0.68-0.80) were generally associated with reduced risk. Similar results were also observed for the other event-specific endpoints (all-cause hospitalizations, CV-2 and CV-3).

\section{DISCUSSION}

There is a general lack of RW data on healthcare management for $\mathrm{CV}$-related comorbidities in patients with T2DM in Germany. There is evidence for several drug classes in terms of their efficacy for managing patients with T2DM and CVD, including high-intensity statins [22, 23], angiotensin-converting enzyme (ACE) inhibitors or angiotensin receptor blockers (ARBs) [24, 25], and SGLT-2is [26] or GLP-1RAs [27]. However, results from smaller studies conducted to date suggest that these agents are consistently underused [28]. The main objective of this study was to describe the RW medication treatment of these patients, to compare the treatment with guideline recommendations for management of CVD and, in an explorative way, to assess whether CV outcomes and mortality are associated with the level of guideline adherence. The main advantage of this analysis is the use of a large claims dataset unaffected by any patient/study site selection bias, including data on outpatient and inpatient treatment on a patient level with a high percentage of continuously observable patients.

As one of the first studies of its kind, our analysis reports numbers on the incidence and prevalence of CVD in patients with T2DM. The majority of patients in our study suffered from diseases like hypertension and dyslipidemia, with more than one-fourth already having had a diagnosis of IS, MI, HF or CAD and facing a substantially increased risk of a CV death. This is reflected by the 12-month mortality after an incident diagnosis of a CVD of interest as high as $22.1 \%$. Both prevalence and incidence numbers as well as mortality emphasize an urgent medical need for the prevention of CV complications after an incident CVD diagnosis.

In high-risk patients with T2DM, intensive intervention with multiple drug combinations and behavioral modifications have shown sustained positive effects. The Steno-2 study [29], in which 160 participants with T2DM randomized to intensive versus conventional control of blood glucose, blood pressure and lipids were followed for an average of 7.8 years, demonstrated that simultaneous management of multiple risk factors synergistically can reduce the risk for a CV event. The results of this study also showed a statistically and clinically significant risk reduction by $53 \%$ (HR $0.47 ; 95 \%$ CI 0.24-0.73) for the composite of death from CV causes, nonfatal MI, nonfatal stroke, revascularization and amputation. While the achievement of multiple treatment goals in diabetes care has improved over time [30], a U.S. study analyzing a survey from 1999 to 2010 demonstrated that still only about one-half of the patients with T2DM are at their recommended personal goals for glycated hemoglobin (HbA1c), blood pressure, and low-density lipoprotein (LDL-cholesterol) [31].

Our study shows a substantial lack of adherence to treatment guidelines in patients with T2DM and incident CVD. As a result, a majority of the patients observed was classified as "yellow" or "red", indicating partial or no agreement with guideline recommendations, and accompanying treatment patterns barely changed in the observed patients between the pre- and post-index periods, using an IS, MI or a diagnosis of $\mathrm{HF}$ or CAD as index event. This underuse of preventive therapies in CAD patients has also been previously demonstrated for 22 European countries in the multicenter, cross-sectional EUROASPIRE study [12] where comprehensive pharmacological therapy was used in only 50\% (CV therapy: 91, 84, 76 and $80 \%$ with use of aspirin, BB, RAAS inhibitors and statins, respectively; AD therapy: 24 and $60 \%$ with use of insulin or oral $\mathrm{AD}$, respectively) of the patients with known diabetes. Another study based on German/Austrian registry data ("Diabetes-Patienten-Verlaufsdokumentation") 
analyzed the achievement of treatment goals in secondary prevention of MI or stroke in patients with T2DM and reported similar findings with even lower prescription prevalence for PAIs (MI 50.7\%; stroke $31.7 \%$ ), statins (MI 57.0\%; stroke $40.1 \%$ ), any type of antihypertensives (MI 65.1\%; stroke $65.8 \%$ ), and ACE inhibitors (MI 49.7\%; stroke 41.3\%) [11]. A recent prospective, pan-European registry study followed a similar approach in investigating guideline compliance differentiated for chronic and acute HF [32], although the study was based on prescription rates. The patients were mainly classified as guideline adherent and had high treatment rates for outpatient use of RAAS inhibitors $(92.2 \%)$, BBs $(92.7 \%)$ and mineralocorticoid receptor/aldosterone antagonist (MRAs) (67.0\%), although far less than one-third of the patients were on the target dosages suggested by the European Society of Cardiology (ESC) guidelines (29.3\% for ACE inhibitors, $24.1 \%$ for ARBs, $17.5 \%$ for $\mathrm{BBs}$, and $30.5 \%$ for MRAs). These results confirm the low guideline adherence-especially for HF-from our analysis. This issue was also addressed in the IMPACT RECO Survey [33], which confirmed under-prescription of HF drugs and observed dosages below treatment goals recommended in guidelines. Additional evidence for lack of concomitant use of CV-related therapies for patients with established atherosclerotic cardiovascular disease (ASCVD) and T2DM was demonstrated in a recent U.S. claims data analysis, which highlighted the underuse of recommended high-intensity statins $(24.7 \%)$ and ACE inhibitors or ARBs (53.1\%) in patients with T2DM. Overall, the authors found that $37.4 \%$ of patients evaluated were not treated with any type of evidence-based therapy to prevent ASCVD [34].

However, low guideline adherence is not necessarily associated with a low quality of care. In the present study, guideline adherence represents the prescription of $\mathrm{CV}$ medication based on billing data from the statutory health insurance funds in patients with T2DM. From these data it is not possible to comment on why the recommended therapies were not prescribed. A recent literature review [35] identified eight studies that examined the relationship between diabetes and being adherent to statin therapy, with the authors suggesting that T2DM is generally associated with higher adherence to treatment guidelines although statins are also known to modestly accelerate the development of diabetes in patients with pre-existing risk factors [36].

The exploratory analysis of our study demonstrated that the lack of guideline adherence with regard to $\mathrm{CV}$ medication therapy is associated with a higher risk of experiencing allcause hospitalizations, pre-specified CV events leading to hospitalization and all-cause death. A similar analysis was performed for HF patients from six European countries (France, Germany, Italy, The Netherlands, Spain and UK) within the prospective MAHLER Survey [37], which assessed $\mathrm{HF}$ and $\mathrm{CV}$ hospitalization rates based on a three-level adherence assessment ("perfect adherence" /"moderate adherence"/"low adherence"). Based on an analysis of data from a 6-month follow-up, 6.7, 9.7 and 14.7\% ("perfect adherence" /"moderate adherence"/"low adherence," respectively) of admissions were due to HF and $11.2,15.9$ and $20.6 \%$, respectively, were due to any other $\mathrm{CV}$ event. These findings are in line with the relatively high event rates identified in our study (admissions due to HF in "fully adherent"/"partly adherent"/"non-adherent" patients: $4.8,12.9$ and $13.3 \%$ and all-cause hospitalization: 21.4, 25.7 and 30.8\%), suggesting that there is still an urgent need to develop and implement stringent quality management programs, such as DMPs. In addition, we showed that diabetes is a significant predictor for time to CV hospitalization (HR 1.43; $P=0.022$ ), underscoring the relevance of implementing appropriate CV management in this patient population. The importance of guideline adherence in reducing $\mathrm{CV}$ risk in patients with T2DM was also confirmed in a Swedish study on lipid-lowering therapies, with the results demonstrating a 44-51\% higher risk of $\mathrm{CV}$ events and a doubled risk of all-cause mortality in patients with low adherence to Swedish lipid-lowering prescribing guidelines [38], despite evidence for increased incidence of T2DM due to statin use [36]. Another U.S. claims data study estimated that 1415 major adverse cardiovascular events (MACE) could be prevented over a 3-year period by closing the 
gap in high-dose statin use in a cohort of patients with T2DM and ASCVD who were currently not adequately treated [34].

We acknowledge that the identified mortality rates reported in our study population are substantially higher than those in respective clinical trials. This might be partly explained by the low adherence to guideline recommendations as demonstrated in our study. In addition, the reported age and comorbidity status of our patients deviates substantially from previous T2DM outcome trials [16-21], which found significant benefits for treatment with SGLT-2is (empagliflozin: EMPA-REG OUTCOME trial; canagliflozin: CANVAS trial) for a reduction in the composite MACE outcome and hospitalizations due to heart failure.

\section{Limitations}

We acknowledge some limitations to our study. First, our conclusions are mainly based on German claims data. The main benefit of such data is access to a large, unselected patient sample which, to the contrary, is also associated with the disadvantage of limited availability of clinical data, patient-reported outcomes and socioeconomic data. Therefore, some disease-/patient-specific characteristics potentially explaining CV risk, such as duration since the first T2DM diagnosis or laboratory values such as HbA1c, LDL-cholesterol or blood pressure could not be evaluated. The cause of death is generally not documented in claims data; consequently we cannot exclude the possibility that all-cause mortality was also influenced by other factors unrelated to CVD. Also, individuals insured by the AOK PLUS represent approximately $5 \%$ of the German population and are geographically restricted to two administrative regions in Germany. Thus, patients insured by AOK PLUS may not fully represent the demographic features of the entire population of patients with T2DM in Germany. However, due to uniform healthcare regulations, data entry requirements and access to health resources, the treatment patterns of AOK PLUS patients are not expected to significantly differ from those of patients in other German regions. In addition, a recent Germany-wide chart review of patients with T2DM and a subsequent comparison of treatment patterns with the AOK PLUS population showed that treatment in the AOK PLUS region does not differ substantially from that overall in Germany [39].

Second, our data are based on patients with T2DM who received an incident diagnosis of a CVD of interest in the given time period. However, most patients in the database had already been diagnosed with one of the target diseases and were therefore excluded from our analysis. Third, our study is limited only to the four most frequent and relevant CVDs in patients with T2DM, namely CAD, HF, MI and IS. Fourth, early death of patients in the groups assigned with no or limited guideline adherence ("red" and "yellow") may have led to the assignment of these patients to these groups, as these patients did not have the opportunity to receive more than one prescription of the respective agent. However, the assignment of patients to the "green" group was even possible based on a single prescription as long as the prescribed dosage reached $>185$ DDDs per patient-year. Fifth, our medication analysis only included medications covered by the sickness fund and our term 'adherence' relates solely to redeemed prescriptions and not to patient's compliance or persistence with respect to target dosages. In the German healthcare market, most CV-specific drugs are only available through physician's prescriptions. However, aspirin (acetylsalicylic acid [ASA]), which is recommended by guidelines for long-term platelet aggregation inhibition after MI or stroke, may be paid by patients out-of-the-pocket as the public price for most packages is below the patient co-pay (minimum $€ 5$ ). Therefore, aspirin is not fully covered in the analyzed claims dataset, although respective out-ofpocket expenses can be considered to be negligible. Nevertheless, in the case of MI and CAD, the use of dual antiplatelet therapy is recommended as a long-term treatment. Therefore, we would still assume to observe at least one of the prescribed PAI agents in the claims database. Furthermore, in Germany, patients can be exempted from co-payments if they reach an annual income-related co-payment threshold, 
which is likely to be the case for a multimorbid patient population with a mean age of 75 years. In these cases, we could even describe the treatment of these patients with ASA and did so in our analyses. Finally, our claims data study can only approximate drug use based on prescription data as documented by German sickness funds. The grace periods we defined in determining the compliance status for agents prescribed during the 12-month observational period (185 DDDs for each drug class recommended in guidelines) generate a relatively conservative estimate but are in accordance with practice documented in the literature $[40,41]$.

\section{CONCLUSIONS}

In our analysis of a large German claims data set of patients with T2DM and an incident major CV disease, we identified a substantial proportion of patients who are not treated in line with existing CV guidelines. A drug treatment not in line with the guidelines was shown to be associated with substantially higher CV event rates as well as a higher mortality risk. We strongly recommend monitoring drug treatment of this population in more detail and potentially including guideline-adherent treatment as an indicator of quality management in healthcare.

\section{ACKNOWLEDGEMENTS}

We thank Sabrina Müller for her contribution to the statistical analysis and the revision of the manuscript.

Funding. The study (including the journal's Rapid Service Fee) was funded by Boehringer Ingelheim Pharma GmbH \& Co. KG, Ingelheim, Germany.

Authorship. All named authors meet the International Committee of Medical Journal Editors (ICMJE) criteria for authorship for this article, take responsibility for the integrity of the work as a whole, and have given their approval for this version to be published.'
Authorship Contributions. MG, SG and TW developed the conception and design of the study. NP organized the database and performed the statistical analysis. LL, JA, ML, MR and SM made substantial contributions to the study design and clinical interpretation of the data. TW and NP wrote the first draft of the manuscript. All authors contributed to manuscript revision, read, and approved the submitted version.

Disclosures. Thomas Wilke participated in this study as a staff member of IPAM and received honoraria from several pharmaceutical/consultancy companies (Novo Nordisk, Abbvie, Merck, GSK, BMS, LEO Pharma, Astra Zeneca, Bayer, Boehringer Ingelheim, Pharmerit). Maximilian Gabler, Silke Geier and Ludwin Ley participated in the steering committee as staff members of Boehringer Ingelheim Pharma GmbH \& Co. KG. Nils Picker participated in this study as staff member of Ingress-Health; the work of Ingress-Health in this study was financed by Boehringer Ingelheim. Jens Aberle, Michael Lehrke, Stephan Martin and Matthias Riedl participated as clinical advisors in the steering committee of this study and received honoraria and reimbursement of travel costs from Boehringer Ingelheim.

Compliance with Ethics Guidelines. Because of the non-interventional, retrospective nature of this study and because our analysis involved an anonymized dataset, neither ethical review nor patients' informed consent was required, in accordance with German law and policies of the institutions assessing patientlevel data (IPAM and AOK PLUS). The study protocol was reviewed and approved by the sickness fund (AOK PLUS) before access to the data was granted. The study was also evaluated by a scientific steering committee to which all the authors belonged and was based on a study protocol approved before the start of data analysis.

Data Availability. The datasets generated and/or analyzed during the current study are not publicly available due to German data protection law for social insurance data (SGB X). 
Open Access. This article is licensed under a Creative Commons Attribution-NonCommercial 4.0 International License, which permits any non-commercial use, sharing, adaptation, distribution and reproduction in any medium or format, as long as you give appropriate credit to the original author(s) and the source, provide a link to the Creative Commons licence, and indicate if changes were made. The images or other third party material in this article are included in the article's Creative Commons licence, unless indicated otherwise in a credit line to the material. If material is not included in the article's Creative Commons licence and your intended use is not permitted by statutory regulation or exceeds the permitted use, you will need to obtain permission directly from the copyright holder. To view a copy of this licence, visit http://creativecommons.org/licenses/by$\mathrm{nc} / 4.0 /$.

\section{REFERENCES}

1. Saeedi P, Petersohn I, Salpea P, et al. Global and regional diabetes prevalence estimates for 2019 and projections for 2030 and 2045: results from the International Diabetes Federation Diabetes Atlas, 9th edition. Diabetes Res Clin Pract. 2019;157: 107843.

2. Rawshani A, Rawshani A, Franzén S, et al. Mortality and cardiovascular disease in type 1 and type 2 diabetes. N Engl J Med. 2017;376(15):1407-18.

3. Wilke T, Groth A, Fuchs A, et al. Real life treatment of diabetes mellitus type 2 patients: an analysis based on a large sample of 394,828 German patients. Diabetes Res Clin Pract. 2014;106(2): 275-85.

4. Narayan KMV, Boyle JP, Thompson TJ, Sorensen SW, Williamson DF. Lifetime risk for diabetes mellitus in the United States. J Am Med Assoc. 2003;290(14):1884-90.

5. Pfeiffer AFH, Klein HH. Therapie des diabetes mellitus typ 2. Dtsch Arztebl Int. 2014;111:69-82.

6. Roffi M, Patrono C, Collet JP, et al. 2015 ESC Guidelines for the management of acute coronary syndromes in patients presenting without persistent st-segment elevation: task force for the management of acute coronary syndromes in patients presenting without persistent ST-segment elevation of the european society of cardiology (ESC), vol. 37. Oxford: Oxford University Press; 2016. p. 267-315.

7. James S, Agewall S, Antunes MJ, et al. 2017 ESC guidelines for the management of acute myocardial infarction in patients presenting with ST-segment elevation. The Task Force for the management of acute myocardial infarction in patients presenting with ST-segment elevation of the European Society of Cardiology (ESC). http://www.escardio.org/ guidelines. Accessed 23 Jan 2020.

8. Kassenärztliche Bundesvereinigung (KBV), VersorgungsLeitlinien (NLV). Nationale VersorgungsLeitlinie Chronische Herzinsuffizienz-Langfassung, 3. Auflage. Version 1. 2019. http://www.akdae.de. Accessed 23 Jan 2020 .

9. Kassenärztliche Bundesvereinigung (KBV), VersorgungsLeitlinien (NLV). Nationale VersorgungsLeitlinie Chronische KHK-Langfassung, 5. Auflage. Version 1. 2019. http://www.akdae.de. Accessed 23 Jan 2020.

10. Endres M, Diener H, Behnke M, et al. S3-Leitlinie: Sekundärprophylaxeischämischer Schlaganfall und transitorische ischämische Attacke (AWMF-Register-Nr. 030/133). 2015. http://www.dgn.org. Accessed 23 Jan 2020.

11. Bohn B, Schöfl C, Zimmer V, et al. Achievement of treatment goals for secondary prevention of myocardial infarction or stroke in 29,325 patients with type 2 diabetes: a German/Austrian DPVmulticenter analysis. Cardiovasc Diabetol. 2016;15: 72. https://doi.org/10.1186/s12933-016-0391-8.

12. Gyberg V, Kotseva K, Dallongeville J, et al. Does pharmacologic treatment in patients with established coronary artery disease and diabetes fulfil guideline recommended targets? A report from the EUROASPIRE III cross-sectional study. Eur J Prev Cardiol. 2015;22(6):753-61.

13. Fricke U, Günther J, Nieprasch-von Dollen K, Zawinell A. Anatomisch-therapeutisch-chemische-Klassifikation mit Tagesdosen Amtliche Fassung des ATC-Index mit DDD-Angaben für Deutschland im Jahre 2019. 2019. Berlin: Wissenschaftliches Institut der $\mathrm{AOK}$

14. Chang HY, Weiner JP, Richards TM, Bleich SN, Segal JB. Validating the adapted diabetes complications severity index in claims data. Am J Manag Care. 2012;18(11):721-6.

15. Charlson M, Szatrowski TP, Peterson J, Gold J. Validation of a combined comorbidity index. J Clin Epidemiol. 1994;47(11):1245-51. 
16. Zinman B, Wanner C, Lachin JM, et al. Empagliflozin, cardiovascular outcomes, and mortality in type 2 diabetes. N Engl J Med. 2015;373(22): 2117-28. https://doi.org/10.1056/NEJMoa1504720.

17. Kato ET, Silverman MG, Mosenzon O, et al. Effect of dapagliflozin on heart failure and mortality in type 2 diabetes mellitus. Circulation. 2019;139(22): 2528-36.

18. Mahaffey KW, Neal B, Perkovic V, et al. Canagliflozin for primary and secondary prevention of cardiovascular events: results from the CANVAS Program (Canagliflozin Cardiovascular Assessment Study). Circulation. 2018;137(4):323-34.

19. White WB, Cannon CP, Heller SR, et al. Alogliptin after acute coronary syndrome in patients with type 2 diabetes. N Engl J Med. 2013;369(14):1327-35.

20. Engel SS, Suryawanshi S, Stevens SR, et al. Safety of sitagliptin in patients with type 2 diabetes and chronic kidney disease: outcomes from TECOS. Diabetes Obes Metab. 2017;19(11):1587-93.

21. Scirica BM, Bhatt DL, Braunwald E, et al. Saxagliptin and cardiovascular outcomes in patients with type 2 diabetes mellitus. N Engl J Med. 2013;369(14): 1317-26.

22. Shepherd J, Barter P, Carmena R, et al. Effect of lowering LDL cholesterol substantially below currently recommended levels in patients with coronary heart disease and diabetes: the treating to new targets (TNT) study. Diabetes Care. 2006;29(6): 1220-6.

23. Baigent C, Blackwell L, Emberson J, et al. Efficacy and safety of more intensive lowering of LDL cholesterol: a meta-analysis of data from 170000 participants in 26 randomised trials. Lancet. 2010;376(9753):1670-81

24. McAlister FA. Angiotensin-converting enzyme inhibitors or angiotensin receptor blockers are beneficial in normotensive atherosclerotic patients: a collaborative meta-analysis of randomized trials. Eur Heart J. 2012;33(4):505-14. https://doi.org/10. 1093/eurheartj/ehr400.

25. Heart Outcomes Prevention Evaluation Study Investigators; Yusuf S, Sleight P, et al. Effects of an angiotensin-converting-enzyme inhibitor, ramipril, on cardiovascular events in high-risk patients. N Engl J Med. 2000;342(3):145-53. https://doi.org/ 10.1056/NEJM200001203420301.

26. Zelniker TA, Wiviott SD, Raz I, et al. SGLT2 inhibitors for primary and secondary prevention of cardiovascular and renal outcomes in type 2 diabetes: a systematic review and meta-analysis of cardiovascular outcome trials. Lancet. 2019;393(10166):31-9.

27. Kristensen SL, Rørth R, Jhund PS, et al. Cardiovascular, mortality, and kidney outcomes with GLP-1 receptor agonists in patients with type 2 diabetes: a systematic review and meta-analysis of cardiovascular outcome trials. Lancet Diabetes Endocrinol. 2019;7(10):776-85.

28. Arnold SV, De Lemos JA, Rosenson RS, et al. Use of guideline-recommended risk reduction strategies among patients with diabetes and atherosclerotic cardiovascular disease insights from getting to an improved understanding of low-density lipoprotein cholesterol and dyslipidemia management (GOULD). Circulation. 2019;140:618-20. https:// doi.org/10.1161/CIRCULATIONAHA.119.041730.

29. Gæde P, Vedel P, Larsen N, Jensen GVH, Parving $\mathrm{H}-\mathrm{H}$, Pedersen O. Multifactorial intervention and cardiovascular disease in patients with type 2 diabetes. N Engl J Med. 2003;348(5):383-93.

30. Saaddine JB, Cadwell B, Gregg EW, et al. Improvements in diabetes processes of care and intermediate outcomes: United States, 1988-2002. Ann Intern Med. 2006;144(7):465-74.

31. Ali MK, Bullard KM, Saaddine JB, Cowie CC, Imperatore G, Gregg EW. Achievement of goals in U.S. Diabetes Care, 1999-2010. N Engl J Med. 2013;368(17):1613-24. https://doi.org/10.1056/ NEJMsa1213829.

32. Maggioni AP, Anker SD, Dahlström U, et al. Are hospitalized or ambulatory patients with heart failure treated in accordance with European Society of Cardiology guidelines? Evidence from 12440 patients of the ESC Heart Failure Long-Term Registry. Eur J Heart Fail. 2013;15(10):1173-84.

33. de Groote P, Isnard R, Assyag P, et al. Is the gap between guidelines and clinical practice in heart failure treatment being filled? Insights from the IMPACT RECO survey. Eur J Heart Fail. 2007;9(12): 1205-11.

34. Nelson AJ, Ardissino M, Haynes K, Shambhu S, Eapen ZJ, McGuire DK, et al. Gaps in evidencebased therapy use in insured patients in the United States with type 2 diabetes mellitus and atherosclerotic cardiovascular disease. J Am Heart Assoc. 2021;10:e016835.

35. Hope HF, Binkley GM, Fenton S, Kitas GD, Verstappen SMM, Symmons DPM. Systematic review of the predictors of statin adherence for the primary prevention of cardiovascular disease. PLoS One. 2019;14(1):e0201196. 
36. Sattar N, Preiss D, Murray HM, et al. Statins and risk of incident diabetes: a collaborative meta-analysis of randomised statin trials. Lancet. 2010;375(9716): 735-42.

37. Komajda M, Lapuerta P, Hermans N, et al. Adherence to guidelines is a predictor of outcome in chronic heart failure: the MAHLER survey. Eur Heart J. 2005;26(16):1653-9.

38. Karlsson SA, Eliasson B, Franzén S, Miftaraj M, Svensson AM, Andersson Sundell K. Risk of cardiovascular event and mortality in relation to refill and guideline adherence to lipid-lowering medications among patients with type 2 diabetes mellitus in Sweden. BMJ Open Diabetes Res Care. 2019;7(1): e000639.
39. Wilke T, Picker N, Mueller S, et al. Real-world insulin therapy in German type 2 diabetes mellitus patients: patient characteristics, treatment patterns, and insulin dosage. Diabetes Metab Syndr Obes. 2019;12:1225-37.

40. Andrade SE, Kahler KH, Frech F, Chan KA. Methods for evaluation of medication adherence and persistence using automated databases. Pharmacoepidemiol Drug Saf. 2006;15:565-74.

41. Haynes RB, Taylor WD, Sackett DL, Gibson ES, Bernholz CD, Mukherjee J. Can simple clinical measurements detect patient noncompliance? Hypertension. 1980;2(6):757-64. 